\title{
Observance Des Mesures Barrières De Lutte Contre La Maladie A \\ Coronavirus-19 \& Morbi-Mortalités Liées Au Cholera En Zone EndemoEpidémique De La République Démocratique Du Congo
}

[Observing Barrier Measures in the Fight against Coronavirus-19 Disease \& Morbidity and Mortality Related To Cholera in Endemoepidemic Health Zone in the Democratic Republic of Congo]

Héman KABEMBA BUKASA* ${ }^{1}$, Teiggy BIRHULA MONGANE ${ }^{2}$, MATALA MATUNGULU JeanMarie $^{3}$, Joseph NDJIBU KAPAFULE $^{4}$

${ }^{1}$ Départementdes sciences Infirmières, Institut supérieur des techniques médicales de Lubao, Lubao, République Démocratiquedu Congo

${ }^{2}$ Economie de santé, Ecole Régionale de Santé Publique, Université Catholique de Bukavu, Bukavu, République Démocratique du Congo

${ }^{3}$ Département l'Enseignement et Administration en Soins Infirmiers, Institut supérieur des techniques médicales de Lubao, Lubao, République Démocratique du Congo

${ }^{4}$ Département des sciences Infirmières, Institut supérieur des techniques médicales de Kalemie, Kalemie, République Démocratique du Congo

\section{RESUME}

Introduction : Le cholera est une maladie diarrhéique hautement contagieuse et en lien étroit avec le déficit de l'observance stricte des mesures d'hygiène. Il constitue un véritable problème de santé publique dans les pays en voie de développement. Cette étude avait pour objectif de déterminer l'impact de l'observance des mesures d'hygiène pendant la période de la maladie à coronavirus-19 sur l'épidémiologie de cholera dans notre milieu.

Méthode : Il s'agit d'une revue documentaire des cas de choléra notifiés et validés durant la période allant de Janvier 2018 à Mars 2021 dans la zone de santéendémo-épidémique de Moba, province du Tanganyika, en République Démocratique du Congo.

Résultats : 1935 cas de choléra ont été rapportés durant la période d'étude, avec respectivement un taux d'attaque pour 100 milles de 196,5 en 2018, 277,2en 2019 et 68,8 en 2020. Le sexe masculin a été le plus affecté en 2018 $(n=368 ; 53,8 \%)$ et 2019 ( $n=527 ; 53,0 \%)$, contrairement avec 2020 en faveur des femmes $(n=153 ; 60,2 \%)$. Les mois pluvieux ont été les plus contributeurs en nombre des cas de choléra notifies. La majorité des malades arrivait avec la déshydratation sévère. Le séjour moyen aux centres de traitement des maladies diarrhéiques (CTMD) et unités de traitement des maladies diarrhéiques (UTMD) a été presqu'uniforme durant la période étudiée, variant de 3,1 à 3,2 jours. Les décès liés au cholera ont été de 1 cas sur 254 en 2020, 19 cas sur 994 en 2019 et 11 cas sur 684 en 2018, soit une létalité de 0,4\% en 2020, 1,9\% en 2019 et 1,6\% en 2018 et une morbidité proportionnelle de $35,4 \%, 51,4 \%$ et $13,1 \%$. 
Conclusion: La limitation des déplacements et l'intensification des mesures d'hygiène instaurées pendant la période de la pandémie de la maladie à coronavirus ont eu un impact significatif sur l'endémicité, l'épidémicité et la mortalité de choléra, spécifiquement le taux d'attaque pour 100 milles et la létalité. En outre, cette période de la maladie à coronavirus expose les femmes à contracter le cholera par rapport à leurs homologues masculins.

MOTS-CLES : Cholera, Coronavirus, Vibriocholerae, Tanganyika, RDC

\section{ABSTRACT}

Background: Cholera is a highly contagious diarrheal disease closely related to the lack of strict observance of hygiene measures. It constitutes a real public health problem in developing countries.

Aim: The objective of this study was to determine the impact of the observation of hygiene measures during the period of coronavirus-19 disease on the epidemiology of cholera in our environment.

Method: This was a documentary review of cholera cases notified and validated during the period from January 2018 to March 2020 in the health zone of Moba (endemo-epidemic), province of Tanganyika, in the Democratic Republic of Congo.

Results: 1,935 cases of cholera were reported from 2018 to 2020 with respectively an attack rate per 100 miles of 196.5 in 2018, 277.2 in 2019 and 68.8. The male sex was the most affected in $2018(n=368 ; 53.8 \%)$ and $2019(n=$ 527; 53.0\%), unlike 2020 in favor of women ( $n=153 ; 60.2 \%)$. The majority of patients arrived with several dehydrations. The average stays at the Diarrheal Diseases Treatment Center (DDTC) and Diarrheal Diseases Treatment units (DDTU) was almost uniform during the study period, ranging from 3.1 to 3.2 days. Cholera-related deaths were 1 case in 2020, 19 cases in 2019 and 11 cases in 2018, i.e. a fatality of $0.4 \%$ in $2020,1.9 \%$ in 2019 and $1.6 \%$ in 2018 and a proportional morbidity of $35.4 \%, 51.4 \%$ and $13.1 \%$.

Conclusion: The hygiene measures introduced during the period of the coronavirus disease pandemic had a significant impact on the endemicity, epidemicity and mortality of cholera, specifically the attack rate per 100 miles and the lethality. Also, this period of coronavirus disease puts women at risk of contracting cholera compared to their male counterparts.

KEYWORDS: Cholera, Coronavirus, Vibrio cholerae, Tanganyika, DRC

\section{INTRODUCTION}

Le cholera est une maladie diarrhéique aigue causée par le Vibriocholerae, une bactérie entero-toxique gram négatif de la famille des Vibrionaceae dont on distingue plusieurs souches [1-4]. Il demeure un sérieux problème de santé publique, surtout dans les pays en voie de développement [1] [5][6]. Le Vibriocholeraeest transmis au travers 1'eau et les aliments souillés par les matièresfécales [7] - [10], d'où l'importance dans l'observance des mesures d'hygiène demeure le plus grand pilier de la lutte contre le choléra [3] [11].

Selon L'organisation mondiale de la santé (OMS), 1,3 à 4 millions de cas de choléra sont notifies chaque année, et le nombre de décès avoisinerait 21 milles à 143 milles [12] [13]. L'Afrique demeure encore à ce jour l'un des continents les plus vulnérable et les plus affecté [8] [14].

La République Démocratique du Congo demeure l'une des zones les plus touchés par le choléra, avec des nombreuses épidémies disséminées dans les pays en 1994 et 2017 [10] [15]. Les foyers les plus importants sont localisés dans les provinces du Nord-Kivu, Sud-Kivu et du Tanganyika (où se trouve le territoire de Moba), et le long du fleuve Congo (Lualaba, Maniema, Kinshasa) [1] [16] - [18]. Le territoire de Moba demeure une zone endémique et épidémique du choléra dont le lac Tanganyika est le facteur naturel de pérennisation [18]. 
Le monde vit en ce $\mathrm{XXI}^{\mathrm{e}}$ siècle au rythme d'une nouvelle épidémie répertoriée depuis Décembre 2019 et causée par un virus ARN monocaténaire linéaire de polarité positive, du genre Beta coronavirus clade B, famille des Coronaviridae : le Coronavirus-19 (appelé au début new coronavirus 2019) [19] [20]. A ce jour, la maladie à coronavirus est une pandémie caractérisée par une forte morbidité et mortalité, associées au désastre économique et psychosocial [20] [21]. Pour freiner sa propagation, nombreuses mesures individuelles et collectives ont été recommandées : limitation des déplacements, distanciation physique, hygiène des mains, etc. [22] - [24]. Ces mesures universelles d'hygiène sont communes au cholera (maladie bactérienne) et la maladie à coronavirus (zoonose virale) [23].

De tout ce qui précède, partant de l'hypothèse admise que l'observance des mesures d'hygiène par la population entrainerait la diminution du nombre des cas des maladies diarrhéiques d'origine hydrique, cette étude a été initié. L'objectif général est de déterminer si les mesures barrières recommandées dans la lutte contre la maladie a coronavirus (Covid19) ont eu un impact sur la morbidité et mortalité de cholera dans notre milieu.

\section{METHODE}

\subsection{Milieu, type et période d'étude}

Il s'agissait d'une revue documentaire de cas de choléra notifiés dans la zone de santé de Moba sur la période allant de Janvier 2018 à Mars 2021. La zone de santé de Moba est située dans territoire administratif de Moba, Province du Tanganyika, au Sud-Est de la République Démocratique du Congo. Elle regroupe 25 aires de santé que nous pouvons repartir en trois axes suivants : axe lac Tanganyika (Kapakwe, Moba-Port, Regeza, Mulunguzi, Kansenge, Liombe, Kizike, Kapampa, Kiku, Kapote, Livua, Moliro), axe centre (Kirungu, Kasama, Mutambala) et axe plateau de «Marungu » (Mulonde, Kasenga, Lumono, Lyapenda, Pepa, Kipiri, Fube, Mwange, Selembe, Musosa). La population vit essentiellement de la pêche sur l'axe lac et de l'agriculture sur les axes centre et plateau. Le climat est tropical et tempéré (plateau de «Marungu »). Deux saisons se succèdent : saison de pluie (Octobre à Mi-Avril) et saison sèche (Avril à Septembre). La densité moyenne de la population est autour de 30 habitants par kilomètre carré. Du point de vue épidémiologique, l'ordre des maladies se présente de la manière suivante : maladies avec grande morbidité (paludisme, malnutrition aigüe sévère, infections respiratoires aigües, diarrhées simples et tuberculose), maladies avec grande mortalité (paludisme, VIH/SIDA, tuberculose, malnutrition aigüe sévère et lèpre), et maladies endémo- épidémiques (Cholera, lèpre, fièvre typhoïde, VIH/SIDA, rougeole) [Source : Plan d'Action Opérationnelle validé de la Zone de santé de Moba 2020 ; www.caid.cd.index.php/donnees-par-provinceadministrative-province-de-tanganyika/territoire-d-moba/?selecteur=fiche consulter le 21/12/2016]. Parmi les problèmes de la zone de santé demeure la faible qualité de soins et services curatifs.

\subsection{Population et paramètres étudiés}

Cette étude couvre sur la population de l'ensemble de la zone de santé de Moba, soit 348102 habitants en 2018, 358544 habitants en 2019, 369301 habitants en 2020 et 380382 habitants en 2021. Tous les cas notifiés de cholera pendant la période d'étude ont été inclus. Les listes linéaires de suivi des cas de choléra avait permis la récolte des données. Les paramètres recherchés étaient : le nombre des cas notifiés et validés sur la période, l'âge, le sexe, le degré de déshydratation a l'admission au centre de traitement des maladies diarrhéiques (CTMD) ou unité de traitement des maladies diarrhéiques (UTMD), le séjour moyen des malades en observation, le nombre de décès. Les cas de choléra ont été validés sur base des signes cliniques classiques et des résultats au test de Cristal (positif ou négatif). 


\subsection{Traitement et analyse statistique des données}

Les données des listes linéaires de cholera de 2018 à 2021 ont été enregistrées sur le fichier Excel (version Microsoft 2010, USA) et analysées. Les résultats obtenus ont été présentés sous forme des tableaux et graphiques. La moyenne, le taux de létalité, le taux d'attaque pour 100 milles et le taux de morbidité proportionnelle ont été calculés. Aucun test statistique de comparaison n'avait été utilisé.

\section{RESULTATS}

Tableau I. Caractérisés évolutives des cas de cholera

\begin{tabular}{|c|c|c|c|c|}
\hline CRITERES & 2018 & 2019 & 2020 & 2021 \\
\hline Population totale $*$ & 348102 & 358544 & 369301 & 380382 \\
\hline Cas & 684 & 994 & 254 & $3 * *$ \\
\hline \multicolumn{5}{|l|}{ Age (ans) } \\
\hline Moyen & 26,8 & 29,2 & 27,5 & \\
\hline Extrêmes & $1-75$ & $1-86$ & $2-81$ & \\
\hline \multicolumn{5}{|l|}{ Sexe } \\
\hline Masculin (\%) & $368(53,8)$ & $527(53,0)$ & $101(39,8)$ & \\
\hline Féminin $(\%)$ & $316(46,2)$ & $467(47,0)$ & $153(60,2)$ & \\
\hline \multicolumn{5}{|l|}{ Déshydratation } \\
\hline $\mathrm{A}(\%)$ & $5(0,7)$ & $4(0,4)$ & $1(0,4)$ & \\
\hline B $(\%)$ & $131(19,2)$ & $291(29,3)$ & $72(28,3)$ & \\
\hline $\mathrm{C}(\%)$ & $548(80,1)$ & $699(70,3)$ & $181(71,3)$ & \\
\hline Séjour moyen (jours) & 3,1 & 3,2 & 3,2 & \\
\hline Décès & 11 & 19 & 1 & \\
\hline Taux d'attaque pour 100000 habitants & 196,5 & 277,2 & 68,8 & \\
\hline Taux de létalité (\%) & 1,6 & 1,9 & 0,4 & \\
\hline Morbidité proportionnelle (\%) & 35,4 & 51,4 & 13,1 & \\
\hline
\end{tabular}

*Population d'accroissement annuel ; **Les quinze premières semaines de l'année 2021

Durant la période de notre étude, 1935 cas de choléra ont été notifiés de Janvier 2018 àMars 2021, soit 684 en 2018 (soit 196,5 pour 100 milles), 994 en 2019 (soit 277,2 pour 100 milles), 254 en 2020 (soit 68,8 pour 100 milles) et 3 cas au premier trimestre 2021. La morbidité proportionnelle a été de $35,4 \%$ en $2018,51,4 \%$ en 2019 et $13,1 \%$ en 2020.(Tableau 1, figure 1)

Les aires de santé de l'axe lac Tanganyika sont quasiment les seules affectées, en dehors de quelques cas sporadiques notifiés dans les aires de santé de Mutambala, Mulonde et Kirungu.

L'âge moyen des malades de cholera dans les CTMD et UTMD a été de 26,8 ans en 2018, 29,2 ans en 2019 et 27,5 ans en 2020 . 


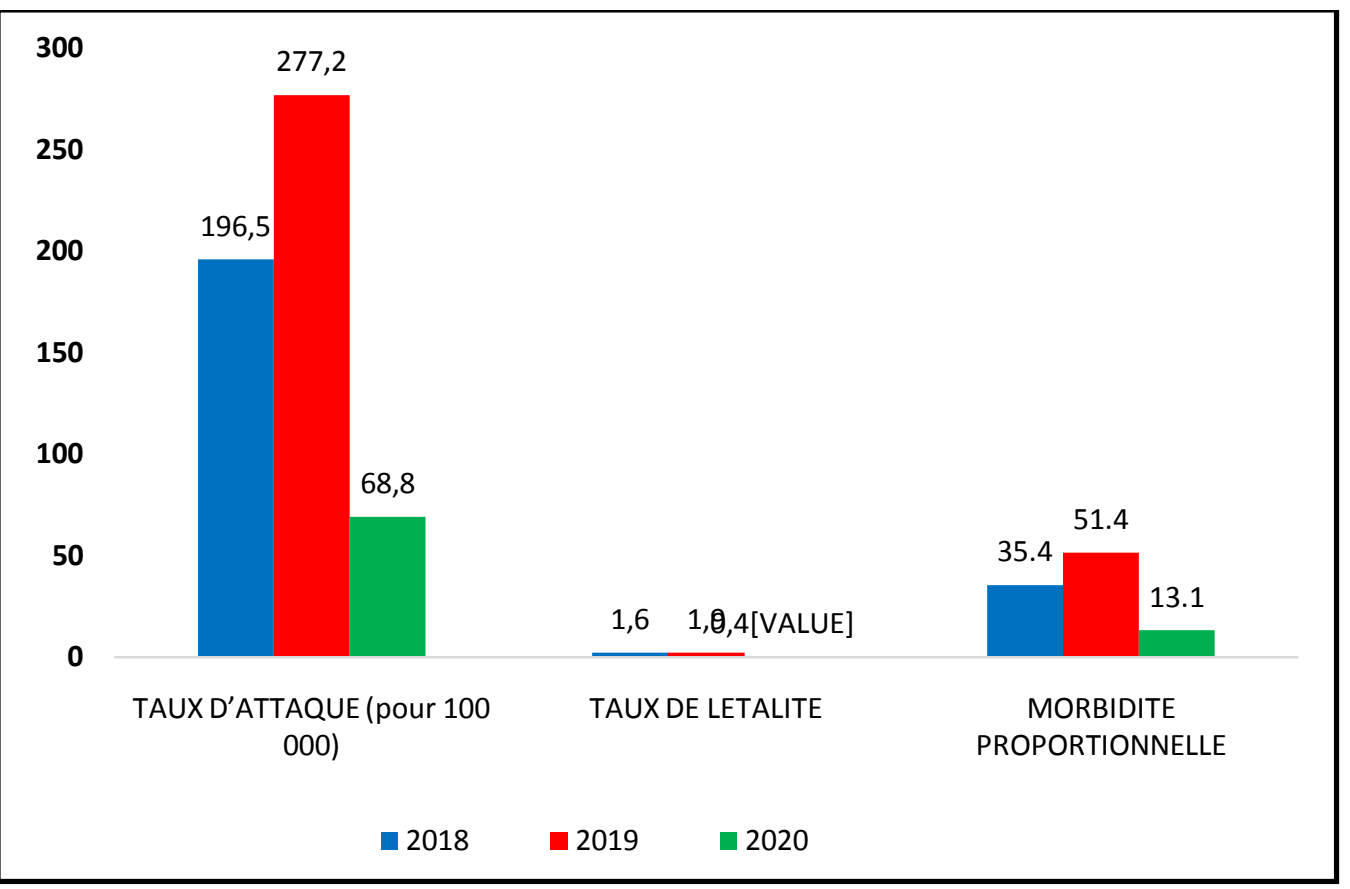

Figure 1. Comparaison de morbidité et mortalité liées au cholera de 2018 à 2020

La majorité des malades était de sexe masculin en 2018 et 2019, soit respectivement 53,8\% (368 cas), et 53,0\% (527 cas). Par contre l'année 2020 avait enregistrée 60,2\% (152 cas sur 254) des malades de sexe féminin.(Tableau 1, figure 1)

La déshydrations sévère nécessitant la réhydratation au plan $\mathrm{C}$ avait été rencontrée chez les malades aux CTMD et UTMD, suivi de plan B et A, soit des déshydratations sévères de l'ordre de 80,1\% (548 cas) en 2018, 70,3\% (699 cas) en 2019 et 71,3\% (181 cas) en 2020. Le séjour moyen des malades aux CTMD et UTMD variait de 3,1 à 3,2 jours. Dans cette série d'étude, le taux de létalité a été de 1,6 \% (11 cas sur 684) en 2018, 1,9\% (19 cas sur 994) en 2019 et $0,4 \%$ (un cas sur 254) en 2020. (Tableau 1, figure 1)

Selon les semaines épidémiologiques, les périodes allant de la semaine 14 à la semaine 25 et de la semaine $37 a ̀ l a$ semaine 52 sont les plus contributrices en terme des cas notifiés de choléra, avec des pic dépassant même 80 cas de choléra par semaine. Il se dégage une particularité allant dela $16^{\mathrm{e}}$ semaine épidémiologique de l'année 2020 jusqu'au premier trimestre 2021 : le nombre des cas notifiés ne dépasse pas deux par semaine et la fréquence des semaines avec zéro cas de choléra augmente. (Figure 2) 
DOI: $\underline{10.51386 / 25815946 / i j s m s-v 4 i 3 p 104}$

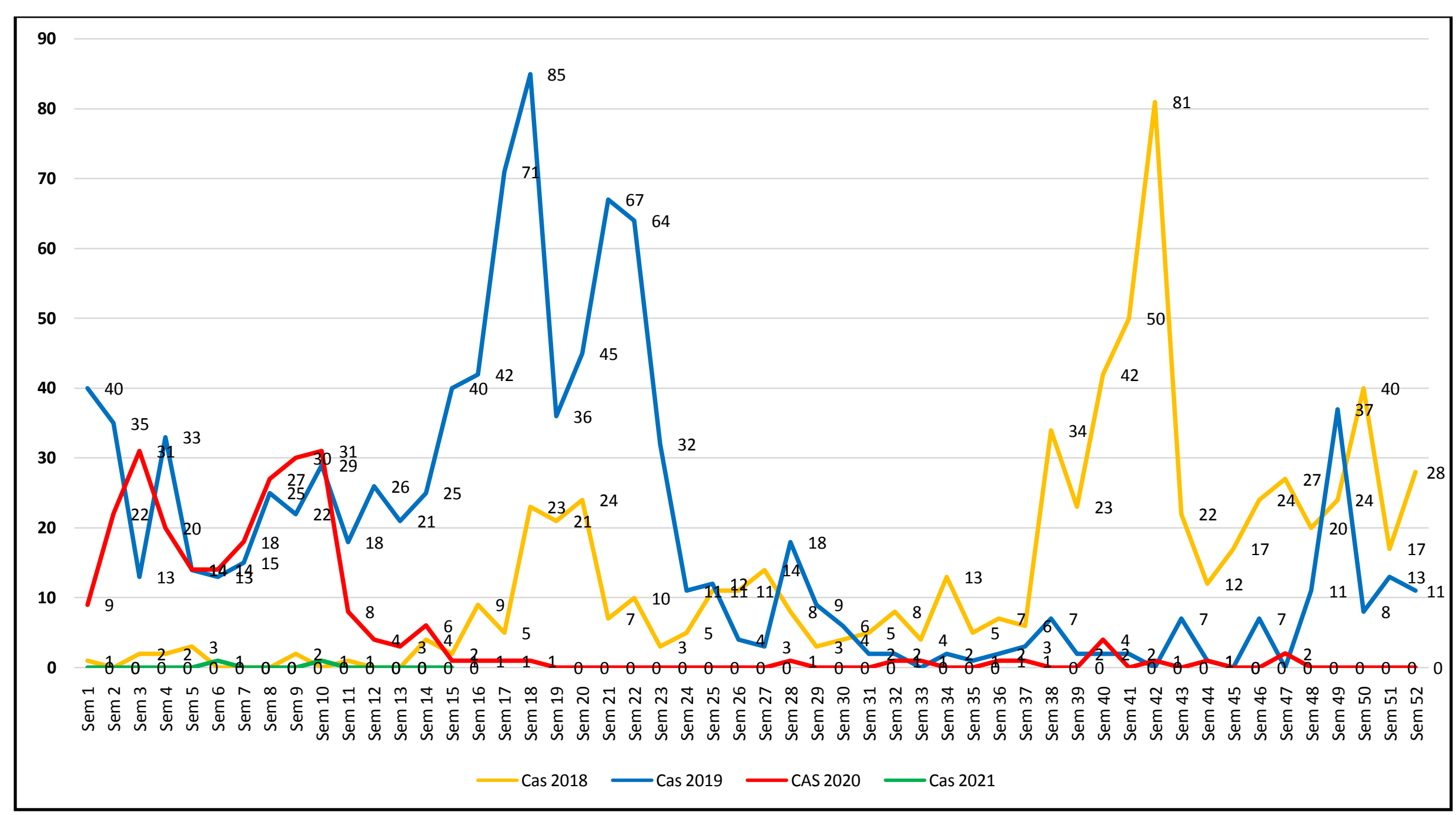

Figure 2. Cas de Choléra notifiés selon les Semaines Epidémiologiques, Janvier 2018 - Mars 2021 


\section{DISCUSSION}

Le cholera est une maladie diarrhéique aigue causée par le VibrioCholerae, et favorisée par un contexte de déficit d'hygiène et de faiblesse dans le système de surveillance et d'alerte des maladies [18] [25] [26]. Parmi les mesures de lutte, un accent particulier doit être réservé à l'adhésion de la population aux mesures d'hygiène et à la fréquentation des structures sanitaires en cas de maladie, l'arrivée tardive étant l'un des grands facteurs favorisant la mortalité [15].

Le sexe masculin a été prédominant parmi les malades de choléra, avant 2020. Par contre, en 2020, le sexe féminin a été plus affecté le choléra. Cette prédominance féminine avait été évoqué par Baluku $\mathrm{M}$ et $a l$. [8] en Ouganda. Nous pensons que la recherche de survie de la famille et les travaux ménagers dont l'intensification de la recherche de l'eau pour les besoins des ménages serait à la base de cette vulnérabilité des femmes. En effet, dans notre milieu et en Afrique en général, les femmes demeurent les maillons essentiels de la survie des ménages, surtout dans un contexte de restriction des déplacements (tropdes rigueurs pour les hommes que les femmes par les forces de sécurité) où les crises financières et économiques ont été évoquées [27] [28].

Les malades arrivent souvent en retard dans un tableau de déshydratation sévère exigeant de réhydratation au plan $\mathrm{C}$ en ayant recours au Ringer lactate. Le même constat avait été évoqué à Lubumbashi (RDC) en 2014 par Cilundika MP et al. [15]. La raison serait le recours à l'automédication.

C'est au Mois de Mars 202 que la RDC a été secouépar la maladieà coronavirus. Dans cette étude, il a été constaté une diminution des cas de choléra depuis cette même période jusqu'à ce jour.

Dans une étude menée à Moba en 2016 [18], il a été démontrée que l'hygiène déficitaire, avec notamment la consommation des eaux du lac Tanganyika et la faible proportion des personnes se lavant les mains après des moments critiques tels qu'au retour des installations hygiéniques (toilettes), était à la base de la persistance du nombre élevé des cas de choléra et des épisodes d'épidémies de cholera.

Malgré les fortes pluviométriesconstatées depuis 2020 et au cours du premier trimestre 2021 dans notre milieu, ce qui favoriserait la dégradation de l'environnement hygiénique et des flambées épidémiques de choléra, les cas des malades de cholera avait drastiquement diminués. Il en est de même de la mortalité attribuable au cholera.

Les aires de santé de l'axe lac Tanganyika sont quasiment les seules contributrices des cas de choléra, surtout celle de Moba-Port. La proximité avec le lac Tanganyika et le recours à ses eaux pour l'alimentation et l'usage domestique constituent des facteurs de risque importants, comme le souligne Shapiroet al. [29], Taylor et al. [9], Hounmanouet al.[30] et Bompangweet al.[10] concernant les lacs Victoria, Nyasa et Tanganyika. Il a été constaté que plus on s'éloigne du lac, la notification des cas de choléra dans le système de surveillance des maladies devient nulle.

Dans une étudepréliminaire sur le choléraàMoba [18], les auteurs avaient démontré que la communication pour le changement de comportement et l'amélioration des conditions d'hygiène demeuraient, comme souligné par certains chercheurs[31] - [33], les piliers de la diminution drastique de la morbidité et de la mortalité liées au cholera. C'est ce qui a été constaté durant la période de la pandémie à covi-19 dans notre milieu.

En effet, la forte médiatisation sur la gravité de la maladie à coronavirus (images des décès dans les pays occidentaux tels que les Etats-Unis, la France, l'Espagne et l'Italie) et les Etats d'urgence sanitaires décrétés dans le pays semblent avoir eu pour impact une forte diminution des cas notifiés de cholera. La limitation des déplacements (rester à domicile et télétravail), la désinfection de l'environnement, le lavage régulier des mains et la distanciation physique ont été parmi les messages véhiculés dans la sensibilisation des communautés [23] [27] [34].

S'il a été admis que l'association covid-19 et cholera entrainerait un grand risque de mortalité [20] suite aux déshydratations sévères, vomissements et difficultés respiratoires, l'observance des mesures individuelles et collectives d'hygiène est salutaire dans le cadre de la prévention. 
Plusieurs observateurs impliqués dans la surveillance épidémiologique craignent une forte morbidité et mortalité suite aux inondations et à la montée des eaux du lac Tanganyika avec mouvements massifs des populations dans un contexte de changement climatique [3] [35]. Dans cette série d'étude, l'observance inhabituelles des mesures d'hygiène et la limitation des mouvements des populations lors des couvre-feux a eu un impact positif sur la morbidité et mortalité de cholera. Cet effet positif est semblable à celui évoque par Tarnoket al.[36], qui souligne la vulnérabilité que la pandémie de la maladie à coronavirus a relevé. Dans notre contexte, les kits hygiéniques de lavage des mains ont été distribués à temps dans les milieux publics.

A Moba, on constate des pics de notification des cas de choléra durant la période de Septembre à Avril. En effet, cette période correspond à la période de dégradation de l'hygiène environnemental, déjà précaire, suite aux pluies [2][9] [37]. Mais dans le conteste de Covid-19, cette règle n'a pas été respectée.

\section{Limites de cette étude}

Dans le cadre de cette étude, les cas évoqués de choléra n'ont pas été confirmés par la culture bactérienne.

\section{CONCLUSION}

Le choléra et la maladie à coronavirus ont comme exigences communes le risque de contamination été le respect des mesures d'hygiènes. Malgré les inondations et le déplacements massifs des populations suite à la montée des eaux du lac Tanganyika, la forte adhésion de la population, dans un contexte de forte médiatisation de la pandémie de la maladie à coronavirus, a eu un impact positif sur la morbidité et mortalité de cholera. Il est donc pertinent de renforcer la mobilisation sociale des communautés à risque, de continuer à adhérer aux bonnes pratiques de respect des mesures d'hygiène élémentaire (lavage des mains, consommation d'eau potable) afin de diminuer l'impact sanitaire des maladies dites des «mains sales».

\section{Remerciements}

Nous tenons à remercier le superviseur eau, hygiène et assainissement de la zone de santé de Moba, Alexis KABEMBA, pour son apport dans la disponibilité des données ayant contribuées à la réalisation de cette étude.

\section{Conflit d'intérêt}

Aucun conflit d'intérêt en rapport avec cette étude, de la conception a la publication.

\section{REFERENCES}

[1] J. Den ,A.Martin Mengel , D.John Clemens. "Epidemiology of cholera", Vaccine 2020, 38: A31-A40 https://doi.org/10.1016/j.vaccine.2019.07.078

[2] S.K. Jameel, M.A. Shafek, A.M. Abdulmohsen, N.S. Mohamed, S.R. Naji and T.T. Mohammed. "The Isolation of Vibrio cholera and Other Enteric Bacteria with Molecular Characterization of Vibrio cholera during the Outbreak of Baghdad/Iraq in 2015", Advances in Microbiology 2016, 6: 699-715. http://dx.doi.org/10.4236/aim.2016.69069

[3] D. Balasubramanian, S. Murcia, C. Brandon Ogbunugafor, R. Gavilan and S. Almagro Moreno. "Cholera dynamics: lessons from an epidemic", Journal of Medical Microbiology 2021 DOI : https://doi.org/10.1099/jmm.0.001298

[4] V. Diabenoandand E. Kamangu. "Vibrio cholerae: General Knowledge and Epidemiological Aspects", Open Access Library Journal 2019 , 6: e5571. https://doi.org/10.4236/oalib.1105571

[5] M. Kumar, and V.L. Sharma, "Outbreak Investigation of Cholera in a Slum of Northern India"Open Journal of Epidemiology 2014, 4, 243-247. http://dx.doi.org/10.4236/ojepi.2014.44031 
[6] K. Mamady and S. Mafoule, "Cholera in Guinea: The Implication for Safe Water Sources and Sanitations"Open Journal of Preventive Medicine 2014, 4, 535-544. http://dx.doi.org/10.4236/ojpm.2014.47064

[7] B.HassanOsama and B.NellumsLaura "Cholera during COVID-19: The forgotten threat for forcibly displaced populations" $E$ ClinicalMedicine2021, 32 ( 100753): 1-2 https://doi.org/10.1016/j.eclinm.2021.100753

[8] M.Baluku, E.Agwu,A.Kasule, ML. Moazzam,"The transmission dynamics of cholera epidemic in Kasese district, Uganda"Special Pathogens Review Journal (SPRJ) 2015, 1(1): 00030-00039

[9] D.L.Taylor, T.M.Kahawita, S. Cairncrossand J.H.J. Ensink, "The Impact of Water, Sanitation and Hygiene Interventions to Control Cholera: A Systematic Review"PLoS ONE 2015, 10, 8: e0135676. http://dx.doi.org/10.1371/journal.pone.0135676

[10] D.K. Bompangue, P. Giraudoux, P.-D. Plisnier, A. MutomboTinda, M. Piarroux, B. Sudreet al., "Dynamics of Cholera Outbreaks in Great Lakes Region of Africa, 1978-2008", Emerging Infectious Diseases 2011, 17 (11): 2026-2034 DOI: http://dx.doi.org/10.3201/eid1711.110170

[11] L. D'Mello-Guyett, K. Greenland, S. Bonneville, R. D'hondt, M. Mashako, A. Gorski et al., "Distribution of hygiene kits during a cholera outbreak in Kasaï-Oriental, Democratic Republic of Congo: a process evaluation”, Conflict and Health 2020, 14 (51): 1-17 https://doi.org/10.1186/s13031-020-00294-w

[12] OMS. Cholera: Principaux repères, 5 février 2021 https://www.who.int/fr/news-room/fact-sheets/detail/cholera

[13] M.A.C. Pietroni, ”Case management of cholera"Vaccine 2020, 38 : A105-A109 https://doi.org/10.1016/j.vaccine.2019.09.098

[14] S. Rebaudet, B. Sudre, B. Faucher, and R. Piarroux, "Environmental Determinants of Cholera Outbreaks in Inland Africa: A Systematic Review of Main Transmission Foci and Propagation Routes”The Journal of Infectious Diseases 2013, 208 (Suppl 1): S46-54

[15] P.CilundikaMulenga, E. Muse Kikuswe, L. LongombeNdjate, A. Nkumballunga , C. MwelwaMupunga, S. Katanga Mumba et al.« Facteurs de létalité du choléra à Lubumbashi dans la Province du Katanga , en République Démocratique du Congo »Revue médicale de Grands Lacs 2014, 3(3): 345- 360 [Article disponible à: http://www.grandlacs-med-journal.com/images/stories/3320142.pdf]

[16] D. Bompangue, S. Moore, N. Taty, B. Impouma , B. Sudre , R. Mandaet al.,"Description of the targeted water supply and hygiene response strategy implemented during the cholera outbreak of 2017-2018 in Kinshasa, DRC",BMC Infectious Diseases 2020, 20:226 https://doi.org/10.1186/s12879-020-4916-0

[17] RDC. Plan Stratégique Multisectoriel d'Elimination du Choléra en République Démocratique du Congo 2018-2022, Ministère du plan, Janvier 2018, 14-25

[18] B.H. Kabemba, A.M. Ntambwe, S.K. Yamutalua, K. Prince, G.A. Yuma, N.N. Michelineet al., « Why the Endemoepidemicity Persistence of Cholera in Moba (DR. of Congo) ?: Preliminary Study in the Health Area of Regeza in 2016"Open Access Library Journal2018, 5: e3500 https://doi.org/10.4236/oalib.1103500

[19] YC. Wu, CS. Chen, YJ. Chan,“The outbreak of COVID-19: An overview” Journal of the Chinese Medical Association 2020, 83 (3): $217-$ 220.

[20] C. Murugaiah, LSL. Constance, H. Al-Talib, M. Nisha, T. Robin, T. Zainazor Tuan Chilek et al."Risk factors of Covid 19 transmission in cholera endemic countries"International Journal of Medical and Biomedical Studies 2020, 4 (6): 82-83 DOI: https://doi.org/10.32553/ijmbs.v4i6.1204

[21] B. LevinAdam, M. Ball Christine, and J. FeatherstonePeter,"From cholera to COVID-19: How pandemics have shaped the development of anaesthesia and intensive care medicine", Anaesthesia and Intensive Care 2020, 0(0) : 1-11 DOI: https://doi.org/10.1177/0310057X20969701https://orcid.org/0000-0002-7449-706X

[22] Kabamba LN, Kabamba MO, Mukonkole JN, Kabyla BL, Luboya ON. Connaissances, attitudes et pratiques des travailleurs des officines privées sur la covid-19 : cas de la commune de Kintambo à Kinshasa. Revue de l'infirmier congolais.2020;4(1):6-8.

[23] GÜNER Rahmet, HASANOĞLU İmran, AKTAŞ Firdevs .COVID-19: Prevention and control measures in community. Turkish Journal of Medical Sciences 2020, 50: 571-577 https://doi.org/10.3906/sag-2004-146

[24] PKM. Jensen, SL. Grant, ML.Perner, ZZ. Hossain,J. Ferdous, R. Sultanaet al.," Historical and contemporary views on cholera transmission: are we repeating past discussions? Can lessons learned from cholera be applied to COVID-19?", Journal of Pathology, Microbiology and Immunology2021, https://doi.org/10.1111/apm.13102 
[25] E.V.Monakhova, A. Ghosh, A. Mutreja, F.-X.Weill, and T. Ramamurthy, "Endemic Cholera in India and Imported Cholera in Russia: What is Common?",Problems of Particularly Dangerous Infections 2020,3:17-26. (In English). DOI: https://doi.org/10.21055/0370-10692020-3-17-26

[26] BY. Hsueh and CM. Waters, "Combating Cholera [version 1; peer review: 2 approved]", F1000Research 2019, 8 (F1000 Faculty Rev) : 589 https://doi.org/10.12688/f1000research.18093.1

[27] Y. Zhou, R. Xu, D. Hu, Y. Yue, Q. Li, J.Xia, "Effects of human mobility restrictions on the spread of COVID-19 in Shenzhen, China: a modelling study using mobile phone data," Lancet Digital Health 2020 2: e417-24

[28] M. Filary-Szczepanik, "The discipline in the times of Cholera”, Global Affairs 2020,6 (3): 243246, DOI: https://doi.org/10.1080/23340460.2020.1842226

[29] L.Shapiro Roger, R. OtienoMuga, M. Adcock Penny, A. Phillips-Howard Penelope, A. Hawley William,L. Kumar, et al., "Transmission of epidemic vibrio choleraeo1 in rural western Kenya associated with drinking water from Lake Victoria: an environmental reservoir for cholera?, " American Journal of Tropical Medicine and Hygiene 1999, 60, 2: 271-276

[30] MG. Hounmanou Yaovi, K. Mølbak, J.Kähler, H. MdegelaRobinson, E. Olsen John and A. Dalsgaard, "Cholera hotspots and surveillance constraints contributing to recurrent epidemics in Tanzania",BMC Research Notes 2019, 12:664 https://doi.org/10.1186/s13104-019$4731-0$

[31] V.T. Sedas, "Influence of Environmental Factors on the Presence of Vibrio cholera in the Marine Environment: A Climate Link,"The Journal of Infection in Developing Countries 2007, 1 : 224-241. https://doi.org/10.3855/jidc.359

[32] D.K.Karaolis, J.A.Johnson, C.C.Bailey, E.C.Boedeker, J.B Kaper,and P.R.A. Reeves, "Vibrio cholerae Pathogenicity Island Associated with Epidemic and Pandemic Strains," Proceedings of the National Academy of Sciences of the United States of America 1998, 95, 31343139. https://doi.org/10.1073/pnas.95.6.3134

[33] R. Ratnayake, F. Finger, W. John Edmunds and F. Checchi, "Early detection of cholera epidemics to support control in fragile states: estimation of delays and potential epidemic sizes,"BMC Medicine 2020, 18:397 https://doi.org/10.1186/s12916-020-01865-7

[34] GF. Acevedo Christian, "From Cholera to COVID-19: A Historical Review of Misinformation during Pandemics",PROGRESSIO Journal on Human Development 2020, 14: 1-17

[35] P.-D. Plisnier, N. Poncelet, C. Cocquyt, H. De Boeck, D. Bompangue, J. Naithaniet al., "Cholera outbreaks at Lake Tanganyika induced by Climate Change? - "CHOLTIC"', Final Report. Brussels: Belgian Science Policy 2015 - 117 p. (Research Programme Science for a Sustainable Development).

[36] A. Tárnok,"The Cholera Epidemics in Hamburg and What to Learn for COVID-19 (SARS-CoV-2)",Cytometry Part A 2020, 97A: 337339 DOI: https://doi.org/10.1002/cyto.a.23999

[37] K. Moise, A-M.Achille, D. Batumbo , B. Bourdeau, S. Rebaudet, G.Lerebourset al., "Impact of patron saint festivities on cholera in three communes in Haiti",BMC Public Health 2020, 20:1490 https://doi.org/10.1186/s12889-020-09601-9 